\title{
Opposite Regulatory Effects of Iron Ions on the In Vitro Catalytic Activities of NarE, the Toxin-like ADP-ribosyltransferase from Neisseria meningitidis
}

Mariangela Del Vecchio ${ }^{1}$ and Enrico Balducci*

${ }^{1}$ Novartis Vaccines and Diagnostics S.r.I, a GlaxoSmithKline Vaccines company Via Fiorentina 1, 53100 Siena, Italy

${ }^{2}$ School of Biosciences and Veterinary Medicine, University of Camerino, via Gentile III da Varano, 62032 Camerino, Italy

\begin{abstract}
NarE, the mono ADP-ribosyltransferase identified in Neisseria meningitidis, catalyzes three enzymatic reactions. NarE transfers a single ADP-ribose unit to guanidine compounds, hydrolyses NAD in nicotinamide and free ADP-ribose, and ADP-ribosylates itself. We have previously shown that NarE contains an iron-sulfur cluster by biophysical and biochemical analyses. The presence of a structured and stable iron-sulfur cluster is essential for ADP-ribosyltransferase but not for NAD-glycohydrolase activity. We report here that ferric, but not ferrous, ions stimulated the ADPribosyltransferase activity. On the contrary ferrous, but not ferric, ions activated NAD-glycohydrolase activity. These iron effects were reversed when enzymatic reactions were run in the presence of the iron-chelator O-phenantroline. In the presence of either ferric or ferrous ions there was an increase of the Vmax both for transferase and NADase activity while the $\mathrm{K}_{m}$ value for NAD was unaltered. The presence of $10 \mathrm{mM} \mathrm{Fe}^{3+}$ increased the ADP-ribosyltransferase activity when we mutated residues not involved in cluster, while is ineffective when residues involved in cluster are mutated. Similar results were obtained with NAD-glycohydrolase activity. The results presented here demonstrate that iron, which plays an important role in metabolism, can modulate NarE activities depending on its oxidation state. This novel observation could be relevant in the context of Neisseria meningitidis infection.
\end{abstract}

Keywords: ADP-ribosylating toxins; NAD; NAD-glycohydrolase; Neisseria meningitidis; Pathogenesis

Abbreviations: NAD: $\beta$-Nicotinamide Adenine Nucleotide; NAM: Nicotinamide; ARTs: ADP-Ribosyltransferases; NADase: NADGlycohydrolase; $\varepsilon$-NAD: $\mathrm{N}^{6}$-Etheno-NAD; IPTG: Isopropyl-1- $\beta$-DThiogalactopyranoside; B-PER: Bacterial Protein Extraction Reagent; PMSF: Phenyl Methyl Sulfonyl Fluoride; Ni-NTA: Nickel-Nitriloacetic Acid Matrix.

\section{Introduction}

Neisseria meningitidis is a Gram-negative opportunistic human specific pathogen, capable of life-threatening infections like meningitis and septicemia [1], responsible for significant worldwide morbidity and mortality $[2,3]$. Most of the bacterial pathogens possess a large number of toxic products, which target and kill host cells. Among these proteins, ADP-ribosylating toxins represent one of the main virulence factors of pathogens. ADP-ribosylating toxins catalyze the enzymatic ADP-ribosylation reaction [4] in which a single ADP-ribose moiety is transferred from NAD onto critical amino-acid residues of specific protein targets with the simultaneous release of nicotinamide (NAM). Physiological substrates of ADP-ribosylating toxins include elongation factor-2 [5,6], GTP-binding proteins [7], actin [8] and members of the antimicrobial peptide family [9]. We have identified an ORF in the $N$. meningitidis genome that codes for the ADP-ribosyltransferase (ART) NarE, which shares the molecular signature and biochemical features with the ART from V. cholerae and E. coli [10]. In analogy with these two toxins, NarE can ADP-ribosylate arginine, hydrolyze NAD in free ADP-ribose and NAM [11], and ADP-ribosylate itself [12]. A recently discovered feature of NarE is the presence of an iron-sulfur center (FeS) that we identified for the first time in a member of the ART family [13]. The iron coordination site has been resolved and the amino acids, two cysteines (C67 and C128) and two histidines (H46 and H57) that bind iron, have been identified [14]. The lack of assembly of the iron-binding site strongly reduced the transferase activity of NarE, while it affected the hydrolase activity only marginally [13]. These observations suggested that the $\mathrm{Fe}-\mathrm{S}$ cluster and iron could have a role in the catalytic mechanism of NarE [13]. It is well established that the success in survival and colonization of $N$. meningitidis depends on its ability to acquire iron from the host [15]. Moreover, iron regulates the expression of a number of genes involved in N. meningitidis virulence [16]. Since NarE contains iron we hypothesized that this metal could modulate the NarE transferase activity. Following this lead, we assessed the effects that iron ions exert on NarE catalytic activities in vitro. While the ADP-ribosyltranferase activity is enhanced by the presence of ferric ions $\left(\mathrm{Fe}^{3+}\right)$, NAD-glycohydrolase (NADase) is enhanced by ferrous ions $\left(\mathrm{Fe}^{2+}\right)$. Kinetic parameters calculated in the presence of $\mathrm{Fe}^{3+}$ or $\mathrm{Fe}^{2+}$ indicated that $\mathrm{Km}$ for NAD remained unaltered while the Vmax relative to transferase or NADase activities consistently increased in the presence of $\mathrm{Fe}^{3+}$ or $\mathrm{Fe}^{2+}$ respectively. The results we have shown previously [13] are expanded in the present study, in which we describe a selective activation of NarE catalytic activities by ferric and ferrous ions.

\section{Material and Methods}

\section{Chemicals}

[adenine-U- ${ }^{14} \mathrm{C}$ ] NAD $(274 \mathrm{mCi} / \mathrm{mmol})$, [carbonyl- ${ }^{14} \mathrm{C}$ ] NAD $(53$ $\mathrm{mCi} / \mathrm{mmol}$ ) were purchased from Amersham; MHAB N45 filter plates from Millipore; isopropyl-1-thio- $\beta$-D-galactopyranoside (IPTG) from Calbiochem; Bradford reagent for protein quantification and Dowex AG1-X2 were purchased from Bio-Rad. Bacterial Protein Extraction

*Corresponding author: Enrico Balducci, School of Biosciences and Veterinary Medicine, University of Camerino, via Gentile III da Varano, 62032 Camerino, Italy, Tel: +39-0737-402710; Fax +39-0737-636216; E-mail: enrico.balducci@unicam.it

Received: August 20, 2015; Accepted: October 28, 2015; Published October 30, 2015

Citation: Del Vecchio M, Balducci E (2015) Opposite Regulatory Effects of Iron lons on the In Vitro Catalytic Activities of NarE, the Toxin-like ADP-ribosyltransferase from Neisseria meningitidis. Biochem Anal Biochem 4: 214. doi:10.4172/21611009.1000214

Copyright: (C) 2015 Del Vecchio M, et al.. This is an open-access article distributed under the terms of the Creative Commons Attribution License, which permits unrestricted use, distribution, and reproduction in any medium, provided the original author and source are credited. 
Reagent (B-PER) was from Pierce while SimplyBlue Safe Stain from Invitrogen. All other reagents used in this study were purchased from Sigma-Aldrich.

\section{Overexpression and purification of recombinant wild type and mutant NarE}

Recombinant NarE was produced growing Escherichia coli BL21 (DE3) transformed with pET21b+ plasmid carrying the narE gene [11] $18 \mathrm{~h}$ at $37^{\circ} \mathrm{C}$. Recombinant NarE was purified as previously described [13]. Briefly, expression of narE gene was induced by addition of $1 \mathrm{mM}$ IPTG. After $3 \mathrm{~h}$ induction at $25^{\circ} \mathrm{C}$, cells were harvested by centrifugation and resuspended in B-PER using a ratio of $10 \mathrm{ml}$ for $3 \mathrm{~g}$ of pellet in the presence of $0.1 \mathrm{mM} \mathrm{MgCl}, 100$ units of DNase I, and $1 \mathrm{mg} / \mathrm{ml}$ of chicken egg lysozyme. The supernatant obtained after centrifugation of the mixture was loaded onto metal nickel-affinity chromatography column (Ni-NTA; Pharmacia; $1.5 \mathrm{ml}$ matrix/liter of culture volume). Bound proteins were eluted with buffer A $(50 \mathrm{mM}$ sodium phosphate buffer $\mathrm{pH} 7.4,300 \mathrm{mM} \mathrm{NaCl}$ ) containing $250 \mathrm{mM}$ imidazole. The purification steps were carried out mainly under anaerobic conditions inside a portable glove box saturated with a mixture of $\mathrm{N}_{2}$ and $\mathrm{H}_{2}$. When the purification was carried out in aerobic conditions, the protein was used shortly after purification, to prevent iron-cluster disruption by air.

\section{Site-directed mutagenesis}

To mutate amino-acids residues of $N$. meningitidis NarE a PCRbased method was used. Oligonucleotides indicated below carrying a single nucleotide mismatch in the underlined positions were used to generate the mutants:

\section{C4S, AGAGGCATTAGTTCCCAACAAGATGAG;}

\section{C67S, TCTATATGACGGATCLTTATATATCTACGA;}

\section{C128S, AGCTGAAGATTCTGGCTGGCTGTATTCCTGAAG;}

\section{C130S, AGCTGAAGATTGTGGCTCETATTCCTGAAG.}

After mutagenesis and DNA sequencing, all of the generated mutants were cloned into pET2 $1 \mathrm{~b}^{+}$, expressed and purified following the procedure previously described [13].

\section{Enzymatic assays}

ADP-ribosyltransferase activity was measured using the standard assay described previously [17]. Briefly, NarE was incubated in a final volume of $0.3 \mathrm{ml}$, containing $50 \mathrm{mM}$ potassium phosphate ( $\mathrm{pH} 7.5$ ), $20 \mathrm{mM}$ agmatine as ADP-ribose acceptor and $0.1 \mathrm{mM}$ [adenine- $\mathrm{U}$ $\left.{ }^{14} \mathrm{C}\right] \mathrm{NAD}(0.05 \mu \mathrm{Ci})$. After incubation at $30^{\circ} \mathrm{C}$, duplicate samples $(100$ $\mu \mathrm{l})$ were applied to $1 \mathrm{ml}$ columns of Dowex AG 1-X2. [adenine $-{ }^{14} \mathrm{C}$ ] ADP-ribosylagmatine was eluted for radioassays with $5 \mathrm{ml}$ of $\mathrm{H}_{2} \mathrm{O}$ and the radioactivity counted in a Packard Top counter. In alternative, the transferase activity of NarE was tested monitoring the enzymatic transfer of ADP-ribose to poly-arginine by a filter plate-based assay as described [18]. Assays were performed in a total volume of $0.3 \mathrm{ml}$ with $0.6 \mathrm{mg}$ of poly-arginine and $0.1 \mathrm{mM}\left[\right.$ carbonyl $\left.-{ }^{14} \mathrm{C}\right] \mathrm{NAD}(0.05$ $\mu \mathrm{Ci})$. After incubation at $30^{\circ} \mathrm{C}$, samples were precipitated with $0.3 \mathrm{ml}$ of $50 \%(\mathrm{w} / \mathrm{v})$ TCA for $30 \mathrm{~min}$ on ice. Samples $(100 \mu \mathrm{l})$ were placed in quadruplicate in single wells of a mixed cellulose esters filter-plate under vacuum conditions. The unreacted [adenine- $\left.\mathrm{U}_{-}{ }^{14} \mathrm{C}\right] \mathrm{NAD}$ was washed out with 10 volumes of $5 \%(\mathrm{w} / \mathrm{v})$ TCA and the incorporated radioactivity measured in a Packard Top counter.

NADase activity was measured with an assay based on NAM release [19]. This assay was carried out in $50 \mathrm{mM}$ potassium phosphate buffer,
$\mathrm{pH} 7.5,0.1 \mathrm{mM}$ [carbonyl $\left.-{ }^{14} \mathrm{C}\right] \mathrm{NAD}(0.05 \mu \mathrm{Ci})$ in a total volume of 0.3 $\mathrm{ml}$. Samples $(100 \mu \mathrm{l})$ after incubation at $30^{\circ} \mathrm{C}$ were applied in duplicate to $1-\mathrm{ml}$ column of Dowex AG1-X2 and ${ }^{14} \mathrm{C}$-nicotinamide eluted with $5 \mathrm{ml}$ of $\mathrm{H}_{2} \mathrm{O}$ for liquid scintillation counting. As an alternate method, NADase activity was evaluated with a fluorimetric assay as described by Barrio [20]. The reaction was started with the appropriate amount of purified NarE in a final volume of $0.7 \mathrm{ml}$ buffered with $20 \mathrm{mM}$ Tris/ $\mathrm{HCl}$ pH 7.0 containing $10 \mu \mathrm{M}$ 1, $\mathrm{N}^{6}$-etheno-NAD ( $\varepsilon$-NAD). Cleavage of $\varepsilon$-NAD, which causes a 10 -fold fluorescence enhancement, was continuously followed at $37^{\circ} \mathrm{C}$ in a Perkin Elmer spectrofluorometer at an emission wavelength of $410 \mathrm{~nm}$ and an excitation of $300 \mathrm{~nm}$. NADase activity was expressed as $\Delta$ relative fluorescence $/ \mathrm{min}$.

Iron effects were assayed in the transferase and NADase standard mixture assays above described with $0-25 \mathrm{mM}$ of the following salts, $\mathrm{Fe}_{2} \mathrm{Cl}_{3}$ and $\mathrm{FeCl}_{3}$. In these experiments the enzyme preparation was extensively dialyzed against $50 \mathrm{mM}$ potassium phosphate buffer $(\mathrm{pH}$ 7.4) before the assay (Figures 1-3).

\section{Determination of kinetic parameters}

To determine the kinetic constants we used the standard radioactive assay above described. Six concentrations of unlabelled NAD were

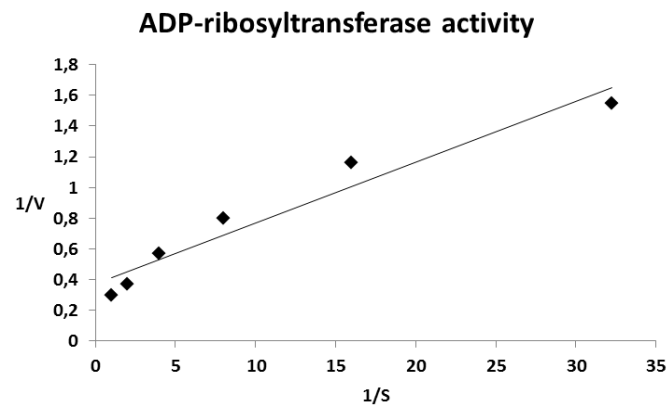

Figure 1: ADP-ribosyltransferase activity.

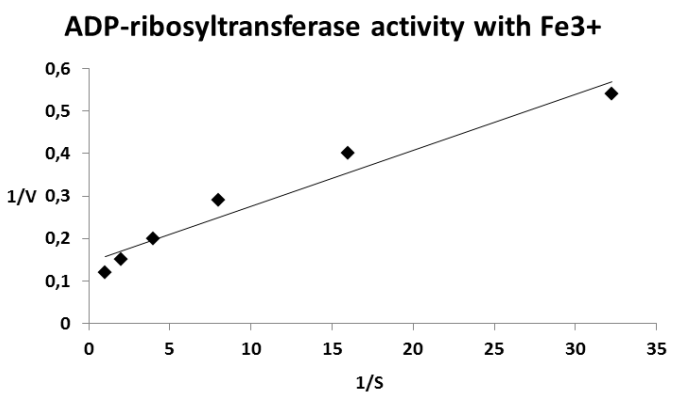

Figure 2: ADP-ribosyltransferase activity with $\mathrm{Fe}^{3+}$.

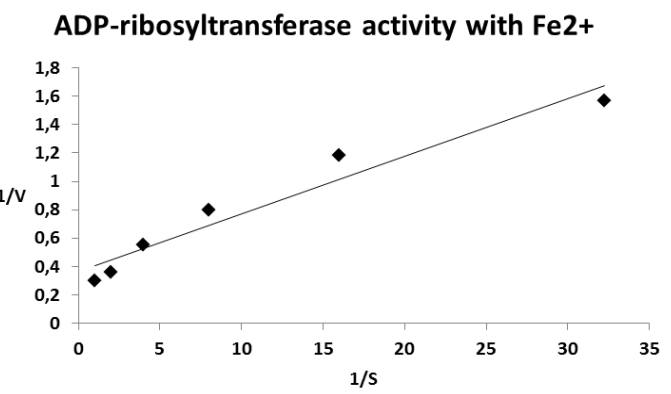

Figure 3: ADP-ribosyltransferase activity with $\mathrm{Fe}^{2+}$. 
added for final concentration of $32.2,16,8,4,2,1$ and $0.5 \mathrm{mM}$. Kinetic parameters were calculated from Lineweaver-Burk plots according to H.I. Segel, Enzyme Kinetics: Behavior and Analysis of Rapid Equilibrium and Steady-state Enzyme Systems. Wiley-Interscience, New York, NY, 1975. Steady-state kinetics was also determined in the presence of 10 $\mathrm{mM} \mathrm{FeCl}{ }_{3}$ or $\mathrm{Fe}_{2} \mathrm{Fe}_{3^{*}}$ The enzyme used for the determination of kinetic analyses was previously dialyzed against $50 \mathrm{mM}$ potassium phosphate buffer.

\section{Protein assay}

Protein concentration was determined using the Bradford assay kit (Bio-Rad) with BSA (Pierce) as a standard [21]

\section{Results}

\section{Ferric ions stimulate NarE transferase activity}

In our recent study we have reported that the ADP-ribosyltransferase of N. meningitidis, NarE, is an iron-containing enzyme [13]. Since the presence of iron in NarE was analyzed using Electron Paramagnetic Resonance spectroscopy (EPR), which identifies paramagnetic metal in proteins, we questioned whether the presence of ferric ions could have some effects on the catalytic activities of NarE. Therefore, we tested the NarE transferase activity in the presence of $10 \mathrm{mM} \mathrm{FeCl}_{3}$ or for comparison, $10 \mathrm{mM} \mathrm{Fe}_{2} \mathrm{Cl}_{3}$. As shown in Figure 4, $\mathrm{Fe}^{3+}$ increased the transferase activity of NarE by 3 fold. This activation was ferricion specific since addition of $10 \mathrm{mM} \mathrm{Fe}_{2} \mathrm{Cl}_{3}$ had no effect. Members of the bacterial ART family such as Cholera and Escherichia coli toxins are activated by $\mathrm{Mg}^{2+}$ ions [22]; therefore, we also performed the experiments in the presence of other trivalent $(\mathrm{Cr}, \mathrm{V})$ and bivalent (Ca, Mn, Li, Co, Ni, Zn, Mg) ions. However, none of the cations used showed any effects (data not shown). Then, we determined the ART activity of NarE with concentrations of $\mathrm{Fe}^{3+}$ ranging from $0.1 \mathrm{mM}$ to $25 \mathrm{mM}$. As shown in Figure 5A, the effect was observed at $5 \mathrm{mM} \mathrm{Fe}^{3+}$ but not at lower concentrations and was more pronounced at 10 and 25 $\mathrm{mM}$ (Figure 5A).

\section{Ferrous ions stimulate NarE NAD-glycohydrolase activity}

Since NarE possesses also NADase activity, we tested whether iron

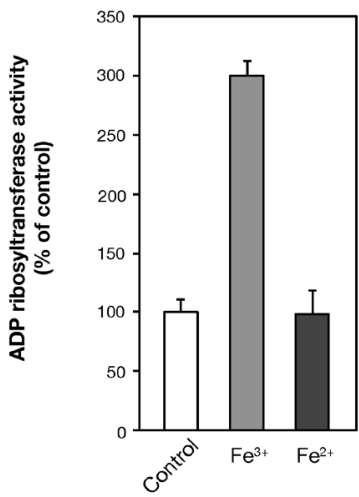

Figure 4: Ferric ion enhances ADP-ribosyltransferase activity. NarE (10 $\mu \mathrm{g})$ extensively dialyzed with $50 \mathrm{mM}$ potassium phosphate buffer $\mathrm{pH} 7.5$ before the assay, was incubated for $18 \mathrm{~h}$ at $30^{\circ} \mathrm{C}$ in $50 \mathrm{mM}$ potassium phosphate buffer, pH 7.5 with $0.1 \mathrm{mM}$ [adenine $-{ }^{14} \mathrm{C}$ ]NAD $(0.05 \mu \mathrm{Ci})$ and with or without (control) $10 \mathrm{mM}$ of $\mathrm{Fe}^{3+}$ or $\mathrm{Fe}^{2+}$ in a total volume of $0.3 \mathrm{ml}$. Samples $(100 \mu \mathrm{l})$ after incubation at $30^{\circ} \mathrm{C}$ were applied to $1-\mathrm{ml}$ column of Dowex AG 1-X2, the ADP-ribosylagmatine formed was eluted with $5 \mathrm{ml}$ of $\mathrm{H}_{2} \mathrm{O}$ for liquid scintillation counting. Activity is expressed as percentage of control. Data shown are the mean $\pm S D$ of three independent assays, with each activity measurement performed in duplicate.
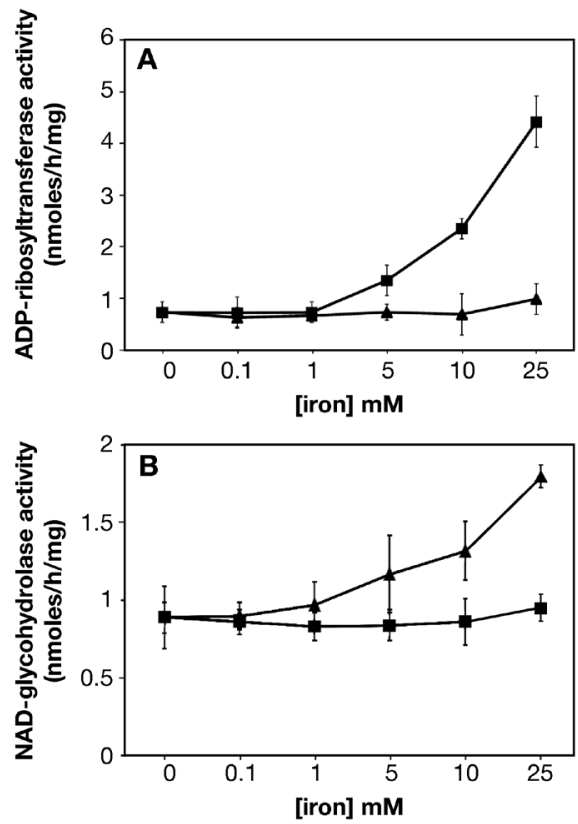

Figure 5: Dose-response curve of ADP-ribosyltransferase and NAD glycohydrolase activities in the presence of iron. (A) Affinity purified NarE was incubated for $18 \mathrm{~h}$ at $30^{\circ} \mathrm{C}$ in $50 \mathrm{mM}$ potassium phosphate buffer with $0.1 \mathrm{mM}$ [adenine-U- $\left.{ }^{14} \mathrm{C}\right] \mathrm{NAD}(0.05 \mu \mathrm{Ci})$ and $20 \mathrm{mM}$ agmatine as ADP-ribose acceptor in the presence of different concentrations of $\mathrm{Fe}^{3+}(\mathbf{\square})$ or different concentrations of $\mathrm{Fe}^{2+}(\mathbf{A})$ as specified in the Figure in a final volume of 0.3 $\mathrm{ml}$. (B) NADase activity was assayed in the same conditions above described in the absence of the ADP-ribose acceptor and with $0.1 \mathrm{mM}$ [carbonyl $-{ }^{14} \mathrm{C}$ ] NAD $(0.05 \mu \mathrm{Ci})$ replacing [adenine-U- ${ }^{14} \mathrm{C}$ ]NAD $(0.05 \mu \mathrm{Ci})$ in the presence of the concentrations of $\mathrm{Fe}^{3+}(\boldsymbol{\Delta})$ or $\mathrm{Fe}^{2+}(/)$ used above in a final volume of $0.3 \mathrm{ml}$. ADP-ribosylagmatine or ${ }^{14} \mathrm{C}$-nicotinamide were purified by AG1-X2 and quantified by scintillation counting. Data are expressed as ADP-ribosylagmatine formed or nicotinamide released per $\mathrm{mg}$ of protein. Data presented are the means \pm SD of three independent assays, with each activity measurement performed in duplicate.

ions affected this enzymatic activity. NADase activity of NarE was unaltered in the presence of various concentrations of $\mathrm{Fe}^{3+}$ (Figure 5B). Of interest $\mathrm{Fe}^{2+}$, which was ineffective on ART activity (Figure $5 \mathrm{~A}$ ), enhanced the NADase activity starting at $5 \mathrm{mM} \mathrm{Fe}^{2+}$ up to 25 $\mathrm{mM}$ (Figure $5 \mathrm{~B}$ ) suggesting an asymmetrical activation of the catalytic activities of NarE. To confirm that the oxidation state of iron has a key role in the regulation of the catalytic activities of NarE, we performed experiments in the presence of $1 \mathrm{mM} \mathrm{Na}_{2} \mathrm{~S}_{2} \mathrm{O}_{4}$ (sodium dithionite), a potent reducing agent. As shown in Table $1, \mathrm{Na}_{2} \mathrm{~S}_{2} \mathrm{O}_{4}$ enhanced the NADase activity while reducing the transferase activity thus reinforcing the hypothesis that the redox state of the iron ions is a key factor for the modulation of the catalytic activities of NarE. No effect on auto-ADPribosylation activity of NarE, were observed at any of conditions and concentrations of $\mathrm{Fe}^{2+}$ and $\mathrm{Fe}^{3+}$ used (data not shown) (Figure 6-8).

\section{The iron-chelating agent $\mathrm{O}$-phenantroline reduces the acti- vating effect of iron}

Both transferase and NADase NarE catalytic activities were assayed by a radioactive assay that implies the use of an anion-exchange resin to separate the unreacted labeled substrate from the product. The anionic interaction of the resin could interfere with the quantification of the enzymatic activities. Therefore, to ensure that our results were not influenced by our analytical method of analysis we assayed NarE transferase activity by using a filter plate-based assay [18] and the NADase activity with a fluorimetric assay [20]. NarE transferase activity 
was increased by the addition of $10 \mathrm{mM} \mathrm{Fe}^{3+}(1.47 \pm 0.21$ vs. $0.49 \pm 0.03$ $\mathrm{nmol} / \mathrm{h} / \mathrm{mg}$ ) while NarE NADase activity was increased by the addition of $10 \mathrm{mM} \mathrm{Fe}^{2+}(0.94 \pm 0.22$ vs. $0.35 \pm 0.15 \Delta \mathrm{F} / \mathrm{min})$ confirming our previous results. To further confirm that the activation of transferase or NADase activity depends on the presence of $\mathrm{Fe}^{3+}$ or $\mathrm{Fe}^{2+}$ in the assay mixture, we used O-phenantroline, which is a specific iron-chelator that decreases NarE transferase activity [13]. As shown in Figure 9A, $\mathrm{O}$-phenantroline decreased the effect of $\mathrm{Fe}^{3+}$ on transferase activity. Similarly the presence of the iron chelator considerably reduced the effect of $\mathrm{Fe}^{2+}$ on NADase activity (Figure 9B), reinforcing the hypothesis of an iron-mediated activation.

\section{Iron enhances maximum velocity of NarE keeping unaltered} affinity for NAD

In order to get insights into the mechanism of iron-mediated

\begin{tabular}{|l|c|}
\hline & ART activity $(\mathbf{n m o l} / \mathbf{h} / \mathbf{m g})$ \\
\hline W/o Na2S2O4 & $0.47 \pm 0.064$ \\
\hline & $0.16 \pm 0.028$ \\
\hline W/o Na2S2O4 & NADase activity $(\mathrm{nmol} / \mathrm{h} / \mathrm{mg})$ \\
\hline Na2S2O4 & $0.35 \pm 0.023$ \\
\hline
\end{tabular}

Table 1: The ADP-ribosyltransferase activity of purified and extensively dialyzed NarE $(13 \mu \mathrm{g})$ was assayed with a filter-based assay. Assays were performed in a total volume of $0.3 \mathrm{ml}$ with $0.6 \mathrm{mg}$ of poly-arginine and $0.1 \mathrm{mM}$ [carbonyl- ${ }^{14} \mathrm{C}$ ]NAD $(0.05$ $\mu \mathrm{Ci}$ ) in the presence of $1 \mathrm{mM} \mathrm{Na} \mathrm{S}_{2} \mathrm{O}_{4}$. Samples of $100 \mu \mathrm{l}$ after incubation at $30^{\circ} \mathrm{C}$ were precipitated with $0.3 \mathrm{ml}$ of $50 \%(\mathrm{w} / \mathrm{v})$ TCA for $30 \mathrm{~min}$ on ice and placed in single wells of a mixed cellulose esters filter-plate under vacuum conditions. To eliminate the unreacted [adenine-U- ${ }^{14} \mathrm{C}$ ]NAD filters were extensively washed with $5 \%(\mathrm{w} / \mathrm{v}) \mathrm{TCA}$ and the incorporated radioactivity measured in a Packard Top counter. Data shown represent the mean $\pm \mathrm{SD}$ of two independent experiments performed in quadruplicate. NADase activity was assayed at $30^{\circ} \mathrm{C}$ for $18 \mathrm{~h}$ with $0.1 \mathrm{mM}$ [carbonyl- ${ }^{14} \mathrm{C}$ ]NAD in a final volume of $0.3 \mathrm{ml}$ in $50 \mathrm{mM}$ potassium phosphate buffer $(\mathrm{pH} 7.5)$ with or without $1 \mathrm{mM}$ $\mathrm{Na}_{2} \mathrm{~S}_{2} \mathrm{O}_{4}$. At the end of incubation $100 \mu \mathrm{l}$ sample were applied to AG1-X2 columns and ${ }^{14} \mathrm{C}$-nicotinamide eluted by water and counted. The values presented represent the means \pm SD of three independent assays.

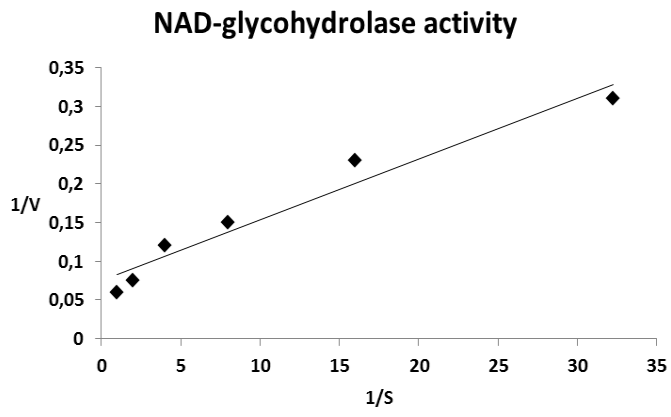

Figure 6: NAD-glycohydrolase activity.

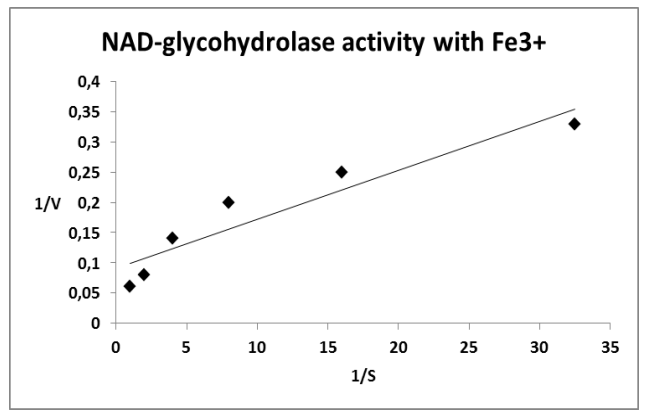

Figure 7: NAD-glycohydrolase activity With $\mathrm{Fe}^{3+}$

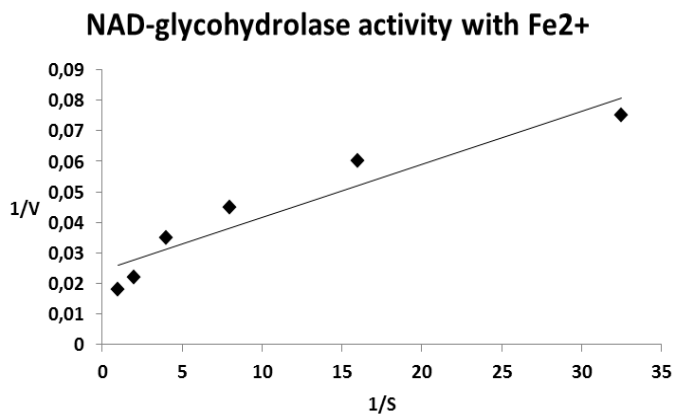

Figure 8: NAD-glycohydrolase activity With $\mathrm{Fe}^{2+}$
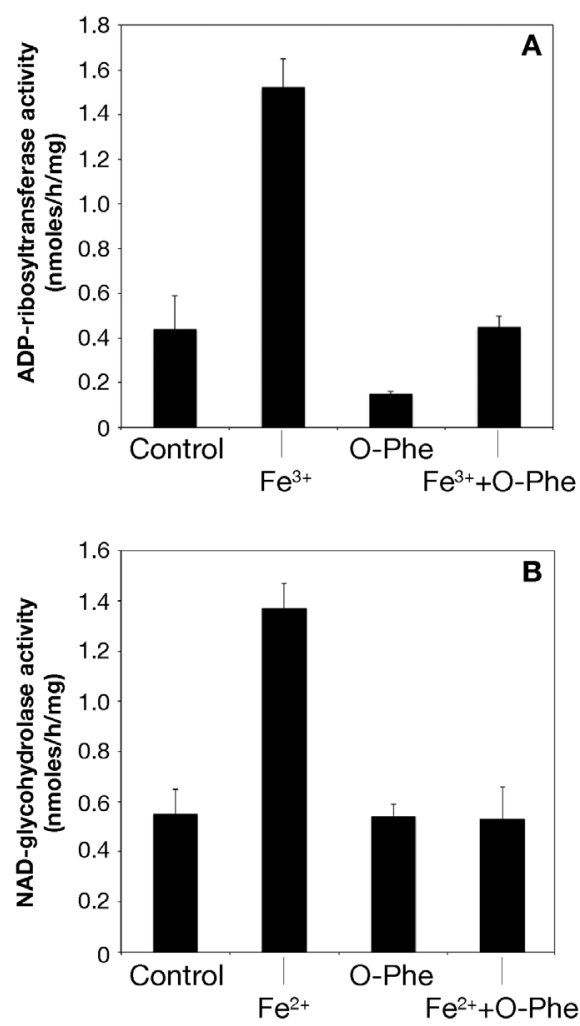

Figure 9: ADP-ribosyltransferase and NAD-glycohydrolase activities in the presence of iron-chelating agent and iron. (A) The ADP-ribosyltransferase activity of purified NarE $(15 \mu \mathrm{g})$ was assayed with a filter-based assay [18] Affinity purified NarE was incubated for $18 \mathrm{hr}$ at $30^{\circ} \mathrm{C}$ with $0.6 \mathrm{mg}$ of polyarginine as acceptor molecule and $0.1 \mathrm{mM}$ [adenine-U- ${ }^{14} \mathrm{C}$ ]NAD $(0.05 \mu \mathrm{Ci})$ in a final volume of $0.3 \mathrm{ml}$ in the presence of $10 \mathrm{mM}$ of $\mathrm{Fe}^{3+}$ with or without $10 \mathrm{mM}$ O-phenantroline. After TCA precipitation, samples $(100 \mu \mathrm{l})$ were applied under vacuum condition in each well of a mixed cellulose ester filter plate and washed with 10 vol. of $5 \%$ TCA. The incorporated radioactivity was measured in a Packard Top counter. Each activity measurement was run in duplicate and analyzed with four replicates. (B) To test NADase activity was used the same enzyme preparation and the radioactive assay described in Experimental procedures. Enzyme activity was assayed at $30^{\circ} \mathrm{C}$ for $18 \mathrm{~h}$ with $0.1 \mathrm{mM}$ [carbonyl $\left.-^{14} \mathrm{C}\right] \mathrm{NAD}$ in a final volume of $0.3 \mathrm{ml}$ in $50 \mathrm{mM}$ potassium phosphate buffer $(\mathrm{pH} 7.5)$ in the presence of $10 \mathrm{mM} \mathrm{Fe}^{2+}$ with or without 10 $\mathrm{mM}$ O-phenantroline. At the end of incubation $100 \mu \mathrm{l}$ sample were applied to $A G 1-X 2$ columns and ${ }^{14} \mathrm{C}$-nicotinamide eluted by water and counted. The values presented represent the means \pm SD of three independent assays.

activation we performed a kinetic analysis of NarE catalytic activities using the radioactive assays. NAD was the variable substrate for both transferase and NADase activities and agmatine the fixed substrate in the transferase reaction. Table 2 lists the kinetic data determined 
for NarE in the absence and in the presence of $10 \mathrm{mM} \mathrm{Fe}^{2+}$ and $\mathrm{Fe}^{3+}$. For comparative purposes, we listed the Vmax ratio compared to the control value. The close $\mathrm{Km}$ values (Table 2 ) indicated that the NAD binding is similar either during the catalysis of the transfer of ADPribose to agmatine used as ADP-ribose acceptor or during the catalysis of NAD hydrolysis to ADP-ribose and NAM. Strikingly, the Vmax values determined increased when iron ions, $\mathrm{Fe}^{3+}$ for transferase and $\mathrm{Fe}^{2+}$ for NADase activity, were added to the assay mixture. The catalytic efficiency with respect to NAD substrate measured as Kcat (turnover number) and ratio between Kcat and $\mathrm{Km}$ indicated a higher efficiency for both transferase and NADase reaction when NarE activities were quantified in the presence of iron.

\section{Role of cluster in iron-dependent activation of NarE catalysis}

We have previously shown that NarE coordinates a single iron ion through C67 and C128, which are involved in the cluster formation and are crucial for transferase activity. We used characterized NarE mutants [13], to assess the relevance of a functionally structured cluster in the iron-mediated activation of NarE. As shown in Figure 10A, the presence of $10 \mathrm{mM} \mathrm{Fe}^{3+}$ increased the transferase activity of the C11S and C130S similarly to that of wt. On the contrary, no activation was detected with the C67S and C128S mutants. In addition, to assess how the conservative mutation influences the ability of NarE to hydrolyze NAD we measured the NADase activity of the four mutants in the presence of $\mathrm{Fe}^{2+}$. As shown in Figure 4B, the NADase activity of C11S and $\mathrm{C} 130 \mathrm{~S}$ was enhanced in the presence of iron. The mutants C67S and C128S, which do not keep a structured cluster but are still able to hydrolyze NAD [13], were not affected by the presence of $10 \mathrm{mM}$ $\mathrm{Fe}^{2+}$ (Figure 10B).

\section{Discussion}

We have previously shown that NarE, a member of the ART family, is an iron-binding protein and the presence of iron is essential for its transferase activity [13]. In this paper we present data showing a selective and iron oxidation-state dependent activation of the catalytic activities of NarE. The action of iron was reversible suggesting that the direct binding of iron leads to the changes of the catalytic properties. The generation of functional information on NarE catalysis is a key point to uncover the pathogenic role of NarE since this protein belongs to the ART family, whose members are usually associated with bacterial pathogenesis [23]. Cytotoxicity exerted by bacterial ARTs is mediated mainly by ADP-ribosylation of target proteins [24]. Besides, bacterial and mammalian ARTs can also possess NADase activity [19],

\begin{tabular}{|l|c|c|c|}
\hline ART activity & $\mathbf{K}_{\mathrm{m}}(\mathbf{m M})$ & $\mathbf{V}_{\max }$ (nmoles/h/mg) & $\begin{array}{c}\mathbf{V}_{\max } \\
\text { compared to control }\end{array}$ \\
\hline- & $0.56 \pm 0.03$ & $5.7 \pm 0.4$ & - \\
\hline $\mathrm{Fe}^{2+}$ & $0.54 \pm 0.09$ & $5.6 \pm 0.4$ & 1 \\
\hline $\mathrm{Fe}^{3+}$ & $0.58 \pm 0.06$ & $18.3 \pm 0.1$ & 3.2 \\
\hline NADase activity & $\mathrm{K}_{\mathrm{m}}(\mathrm{mM})$ & $\mathrm{V}_{\max }(\mathrm{nmoles} / \mathrm{h} / \mathrm{mg})$ & $\begin{array}{c}\mathrm{V}_{\max } \\
\text { compared to control }\end{array}$ \\
\hline- & $0.48 \pm 0.01$ & $22.9 \pm 0.2$ & - \\
\hline $\mathrm{Fe}^{2+}$ & $0.45 \pm 0.02$ & $63.4 \pm 0.3$ & 2.8 \\
\hline $\mathrm{Fe}^{3+}$ & $0.45 \pm 0.03$ & $23.1 \pm 0.3$ & 1 \\
\hline
\end{tabular}

Table 2: $\mathrm{K}_{\mathrm{m}}$ and $\mathrm{V}_{\max }$ values of NarE were determined using $30 \mu \mathrm{g}$ of NarE and 20 $\mathrm{mM}$ of agmatine as ADP-ribose acceptor and variable $\operatorname{NAD}(32.2,16,8,4,2$ and 1 $\mathrm{mM}$ ) for $18 \mathrm{~h}$ at $30^{\circ} \mathrm{C}$ for the transferase activity in the presence or $\mathrm{FeCl}_{3}$ as indicated in the figure. Kinetic parameters for NADase were determined as described above omitting agmatine from the standard assay in the presence of $10 \mathrm{mM} \mathrm{Fe} \mathrm{Cl}_{2}$ Kinetic parameters were calculated from Lineweaver-Burk plots according to $\mathrm{H}$.I. Segel, Enzyme Kinetics: Behavior and Analysis of Rapid Equilibrium and Steadystate Enzyme Systems. Wiley-Interscience, New York, NY, 1975. Data represent the mean $\pm S D$ of three independent experiments performed in duplicate.
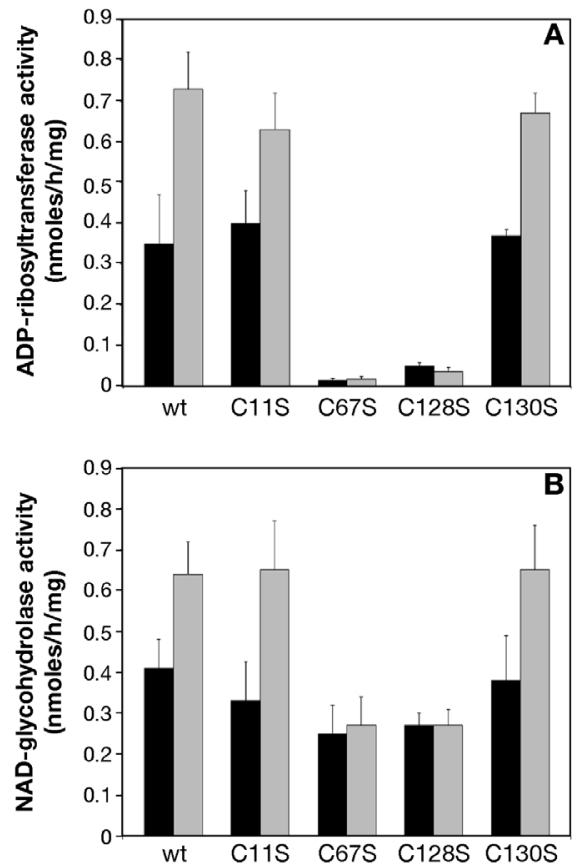

Figure 10: Effect of iron on the ADP-ribosyltransferase and NAD-glycohydrolase activities of NarE mutants. NarE mutants were tested for their ability to transfer ADP-ribose moiety and release NAM with (grey bars) and without (black bars) iron. (A) ADP-ribosyltransferase activity was measured for $18 \mathrm{~h}$ at $30^{\circ} \mathrm{C}$ under the same conditions of previous experiments and as described under Experimental procedures in the presence of $10 \mathrm{mM} \mathrm{Fe}^{3+}$. (B) NADase was quantified with radioactive assay previously described in the same condition used for transferase activity in the presence of $10 \mathrm{mM} \mathrm{Fe}^{2+}$. Data are expressed as ADP-ribosylagmatine formed or NAM release per $\mathrm{mg}$ of purified protein Error bars represent the standard deviation of three independent experiments.

whose physiological function is not clear, and auto-ADP-ribosylation activity [25]. Since in our previous study we identified a paramagnetic iron in NarE, we hypothesized that $\mathrm{Fe}^{3+}$ could also have a role in the regulation of NarE activities. In agreement with this hypothesis, $\mathrm{Fe}^{3+}$ but not $\mathrm{Fe}^{2+}$ induced a concentration-dependent increase in the ART activity. In metal binding sites containing cysteines and histidines $\mathrm{Zn}^{2+}$ can overlap iron, as demonstrated by modeling experiments [14]. Therefore, in addition to other cations including $\mathrm{Mg}^{2+}$, which have positive effects on bacterial ARTs $[22,26]$, we paid particular attention to $\mathrm{Zn}^{2+}$. However, the many experiments carried out in the presence of $\mathrm{Zn}^{2+}$ in different experimental conditions showed no activating effects, while this cation at any concentrations used, precipitated NarE (data not shown). The reason for this precipitation effect is unclear however, since NarE natively binds iron $\mathrm{Zn}^{2+}$ ions could be not physiologically relevant. Of interest and somewhat unexpectedly, the addition of $\mathrm{Fe}^{2+}$ to assay mixture induced an activating effect on glycohydrolase but not on transferase activity (Figure 5). Since we commonly use an anion exchange column to separate the radioactive product from the unreacted substrate, we were concerned that the activation effect could be an artifact. However, this was not the case since alternative assays, which do not imply the use of ionic interactions, gave identical results. The velocity of an enzyme-catalyzed reaction could be influenced by an increased affinity of the enzyme for the substrate or by an increased concentration of active enzyme. In the case of NarE the presence of iron enhanced the $\mathrm{V}_{\max }$ for both catalytic activities without affecting the $\mathrm{K}_{\mathrm{m}}$ for NAD indicating that the presence of the cation increases the concentration of a catalytically active form of NarE. This latter was reinforced by values of Kcat and ratio of Kcat and $\mathrm{Km}$ that indicated 
a higher efficient enzyme. Site-directed mutagenesis and structural studies have shown the strong influence of C67 and C128 substitution in transferase activity and $\mathrm{Fe}^{3+}$ was unable to enhance the activity. These two mutants exhibited only modest reduction of NADase activity but the addition of $\mathrm{Fe}^{2+}$ to the assay mixture failed to enhance the NADglycohydrolase activity. This latter observation suggests that the presence of a cluster coordinated with iron ions is necessary to exert the activating effects both of the transferase and NADase activities. A multistep process involving different proteins is necessary to integrate clusters into proteins [27], in bacteria iron-sulfur clusters due to their sensitivity act as regulators of iron availability allowing bacteria to modify their gene expression profile in response to modified environmental conditions [28]. Moreover, clusters have also a key role in determining the structure and stability of a protein. In this paper we show that they can influence the enzymatic activity of a protein. The modification of the redox state of the iron as it occurs with the addition of $\mathrm{Fe}^{2+}$ or in the presence of a reducing agent that reduces the iron already present in the cluster, likely alters the structure of the protein impeding the access of the ADP-ribose acceptor forcing the enzyme to transfer the ADP-ribose to water. Although the pathogenetic effects of ADP-ribosylating toxins are associated mainly with their transferase activity, toxic effects could also be linked to NADase activity as it occurs in Group A streptococci (GAS) where the toxin possess NADase activity [29]. Iron is a necessary element for survival of $N$. meningitidis into the host and is implicated in the regulation of the expression of a number of bacterial genes involved not only in metabolism but also in virulence [30].

\section{Conclusions}

The direct involvement of iron as regulator of NarE catalytic activities, as shown here appears to be a regulatory mechanism that could provide the missing link in the cellular control of the catalytic activities of NarE. This novel observation can open new possibilities in the participation of this enzyme to $N$. meningitidis physiology. $N$. meningitidis possesses a great ability to colonize different niches in the human body, characterized either by reducing or oxidizing conditions. Therefore, the ability of NarE to modulate its catalytic activities according to the redox state of iron could be instrumental for $N$. Meningitidis pathogenesis.

\section{Acknowledgements.}

Special thanks go to Giorgio Corsi for his precious artwork, to Elisabetta Soldaini for helpful discussion, valuable advice and assistance and Fabio Concetti for technical support. Novartis Vaccines \& Diagnostics, a GlaxoSmithKline Vaccines company financially supported the work.

\section{References}

1. Johnson AP (1983) The pathogenic potential of commensal species of Neisseria. J Clin Pathol 36: 213-223.

2. Schwartz B, Moore PS, Broome CV (1989) Global epidemiology of meningococcal disease. Clin Microbiol Rev 2: 118-124.

3. Schuchat A, Robinson K, Wenger JD, Harrison LH, Farley M, et al. (1997) Bacterial meningitis in the United States in 1995. Active Surveillance Team. N Engl J Med 337: 970-976.

4. Ueda K, Hayaishi O (1985) ADP-ribosylation. Annu Rev Biochem 54: 73-100.

5. Honjo T, Nishizuka $Y$ \& Hayaishi O (1968) Diphtheria toxin-dependent adenosine diphosphate ribosylation of aminoacyl transferase II and inhibition of protein synthesis. J Biol Chem 24: 3553-3555.

6. Iglewski BH, Kabat D (1975) NAD-dependent inhibition of protein synthesis by Pseudomonas aeruginosa toxin,. Proc Natl Acad Sci U S A 72: 2284-2288.

7. Locht C, Keith JM (1986) Pertussis toxin gene: nucleotide sequence and genetic organization. Science 232: 1258-1264.
8. Aktories K, Wegner A (1989) ADP-ribosylation of actin by clostridial toxins. J Cell Biol 109: 1385-1387.

9. Castagnini M, Picchianti M, Talluri E, Biagini M, Del Vecchio M, et al. (2012) Arginine-specific mono ADP-ribosylation in vitro of antimicrobial peptides by ADP-ribosylating toxins. PLoS One 7: e41417.

10. Masignani V, Balducci E, Serruto D, Veggi D, Aricò B, et al. (2004) In silico identification of novel bacterial ADP-ribosyltransferases. Int J Med Microbio 293: 471-478.

11. Masignani V, Balducci E, Di Marcello F, Savino S, Serruto D, et al. (2003) NarE: a novel ADP-ribosyltransferase from Neisseria meningitidis. Mol Microbiol 50: 1055-1067.

12. Picchianti M, Del Vecchio M, Di Marcello F, Biagini M, Veggi D, et al. (2013) Auto ADP-ribosylation of NarE, a Neisseria meningitidis ADP-ribosyltransferase, regulates its catalytic activities. FASEB J 27: 4723-4730.

13. Del Vecchio M, Pogni R, Baratto MC, Nobbs A, Rappuoli R, et al. (2009) Identification of an iron-sulfur cluster that modulates the enzymatic activity in NarE, a Neisseria meningitidis ADP-ribosyltransferase. J Biol Chem 284 33040-33047.

14. Koehler C, Carlier L, Veggi D, Balducci E, Di Marcello F, et al. (2011) Structural and biochemical characterization of NarE, an iron-containing ADPribosyltransferase from Neisseria meningitidis. J Biol Chem 286: 14842-14851.

15. Rohde KH, Dyer DW (2003) Mechanisms of iron acquisition by the human pathogens Neisseria meningitidis and Neisseria gonorrhoeae. Front Biosci 8: d1186-1218.

16. Chen CY, Genco CA, Rock JP, Morse SA (1989) Physiology and metabolism of Neisseria gonorrhoeae and Neisseria meningitidis: implications for pathogenesis. Clin Microbiol Rev 2 Suppl: S35-40.

17. Moss J, Stanley SJ (1981) Histone-dependent and histone-independent forms of an ADP-ribosyltransferase from human and turkey erythrocytes. Proc Nat Acad Sci U S A 78: 4809-4812.

18. Balducci E (2005) A filter plate-based assay for mono ADP-ribosyltransferases [corrected]. Anal Biochem 344: 278-280.

19. Moss J, Manganiello VC, Vaughan M (1976) Hydrolysis of nicotinamide adenine dinucleotide by choleragen and its A protomer: possible role in the activation of adenylate cyclase. Proc Natl Acad Sci U S A 73: 4424-4427.

20. Barrio JR, Secrist JA 3rd, Leonard NJ (1972) A fluorescent analog of nicotinamide adenine dinucleotide. Proc Natl Acad Sci U S A 69: 2039-2042.

21. Bradford MM (1976) A rapid and sensitive method for the quantitation of microgram quantities of protein utilizing the principle of protein-dye binding. Anal Biochem 72: 248-254.

22. Moss J, Stanley SJ, Burns DL, Hsia JA, Yost DA, et al. (1983) Activation by thiol of the latent NAD glycohydrolase and ADP-ribosyltransferase activities of Bordetella pertussis toxin (islet-activating protein). J Biol Chem 258: 11879-11882.

23. Wreggett KA (1986) Bacterial toxins and the role of ADP-ribosylation. J Recept Res 6: 95-126.

24. Glowacki G, Braren R, Firner K, Nissen M, Kühl M, et al. (2002) The family of toxin-related ecto-ADP-ribosyltransferases in humans and the mouse. Protein Sci 11: 1657-1670.

25. Weng B, Thompson WC, Kim HJ, Levine RL, Moss J (1999) Modification of the ADP-ribosyltransferase and NAD glycohydrolase activities of a mammalian transferase (ADP-ribosyltransferase 5 ) by auto-ADP-ribosylation. J Biol Chem 274: 31797-31803.

26. Moss J, Stanley SJ, Morin JE, Dixon JE (1980) Activation of choleragen by thiol: protein disulfide oxidoreductase. J Biol Chem 255: 11085-11087.

27. Beinert H, Holm RH, Münck E (1997) Iron-sulfur clusters: nature's modular, multipurpose structures. Science 277: 653-659.

28. Miller HK, Auerbuch V (2015) Bacterial iron-sulfur cluster sensors in mammalian pathogens. Metallomics 7: 943-956.

29. Bricker AL, Carey VJ, Wessels MR (2005) Role of NADase in virulence in experimental invasive group A streptococcal infection. Infect Immun 73: 6562 6566.

30. Perkins-Balding D, Ratliff-Griffin M, Stojiljkovic I (2004) Iron transport systems in Neisseria meningitidis. Microbiol Mol Biol Rev 68: 154-171. 